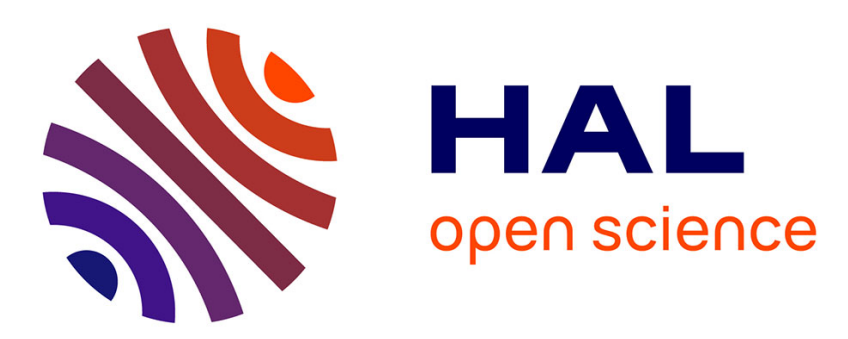

\title{
Conformational Changes and Charge Transfer in Biomolecules Resolved Using Dynamic Enhanced Raman Correlation Spectroscopy
}

Aymeric Leray, Jean-Emmanuel CleMent, Alexandre Bouhelier, Eric Finot

\section{- To cite this version:}

Aymeric Leray, Jean-Emmanuel CleMent, Alexandre Bouhelier, Eric Finot. Conformational Changes and Charge Transfer in Biomolecules Resolved Using Dynamic Enhanced Raman Correlation Spectroscopy. Journal of Physical Chemistry B, 2019, 123 (9), pp.1931-1938. 10.1021/acs.jpcb.8b10803 . hal-02322742

\section{HAL Id: hal-02322742 \\ https://hal.science/hal-02322742}

Submitted on 13 Jan 2022

HAL is a multi-disciplinary open access archive for the deposit and dissemination of scientific research documents, whether they are published or not. The documents may come from teaching and research institutions in France or abroad, or from public or private research centers.
L'archive ouverte pluridisciplinaire HAL, est destinée au dépôt et à la diffusion de documents scientifiques de niveau recherche, publiés ou non, émanant des établissements d'enseignement et de recherche français ou étrangers, des laboratoires publics ou privés. 


\section{Conformational Changes and Charge Transfer}

in Biomolecules Resolved Using Dynamic

\section{Enhanced Raman Correlation Spectroscopy}

Aymeric Leray, ${ }^{*}$ Jean-Emmanuel Clément, Alexandre Bouhélier, and Eric Finot*

Laboratoire Interdisciplinaire Carnot de Bourgogne, UMR 6303 CNRS, Université de

Bourgogne Franche Comté, F-21078,Dijon, France

E-mail: aymeric.leray@u-bourgogne.fr; eric.finot@u-bourgogne.fr 


\begin{abstract}
In this contribution, we report that conformational changes of molecules which are often buried in a wide-distributed Gaussian distribution can be discerned by analysing the dynamics of specific Raman lines. We investigate the pertinence of the auto and cross-correlations functions applied to the dynamics of three Raman lines of an aminoacid, the tryptophan. The cross-correlation between intensity and Raman band is an indicator of the charge transfer during the diffusion limited reaction of tryptophan and gold surface. The Péclet number $P e$ can provide a valuable indicator of the convective and/or diffusive features of each Raman band. Adsorption induced conformation changes can be identified using the auto-correlation of the multiples states within the Raman band centered at $1550 \mathrm{~cm}^{-1}$.
\end{abstract}

\title{
INTRODUCTION
}

From a biochemical point of view, understanding the structure-properties of a macromolecule requires the examination of molecular conformations. The most probable molecules conformations can be currently envisaged by molecular dynamics simulations. However, the experimental verification of these states is still a challenging issue. The primary methods for identifying the structure of crystallized proteins relie on X-ray scattering and NMR spectroscopy but they do not provide any kinetic information. Optical techniques based on fluorescence such as FRET (Förster Resonance energy transfer ${ }^{1,2}$ ) or circular dichroism ${ }^{3}$ are amenable to kinetic studies but are limited to secondary structures or require strategic biomodification using fluorophore localized on appropriate domain.

In this work, we examine the relevance of Raman enhanced spectroscopy for identification of conformational changes in biomolecules. High concentration Raman spectroscopic studies have been first used to determine the molecular composition by identifying Raman peaks from Raman spectral libraries. Advances in surface enhanced Raman spectroscopy (SERS) now make it possible to probe single molecules vibrational interactions in a diluted 
medium. ${ }^{4-6}$ When investigating single molecule, large spectral and temporal fluctuations with abrupt modifications in both intensity $I$ and Raman shift $\nu$ of SERS spectra have already been reported. ${ }^{7-13}$ The observed fluctuations give an unequaled spectral wealth and open a promising way to examine the conformations of biomolecules. However, these SERS fluctuations are not totally exploited because they derive from multiple sources:

- Fluctuations of the Electro-Magnetic (EM) field enhanced by a factor of $10^{4}$ up to $10^{8}$ are mainly explained by the localized surface plasmon resonance. ${ }^{14}$ Intensity fluctuations in the laser radiation (time decay of $0.1 \mu \mathrm{s}$ ) may be also at the origin of these EM field fluctuations. ${ }^{9}$

- Various orientation (or binding) of the adsorbate on the metallic surface may be also at the origin of fluctuations. Large spectral shifts have been reported experimentally and theoretically that have been explained by the interaction between the molecule and the surface. ${ }^{15-18}$

- The chemisorption of a molecule onto a surface is the slowest process achievable experimentally with decay time ranging between 1 s (ionic interaction) and $1 \mathrm{~h}$ (covalent bond). During chemisorption, an electron can be transferred from the metal to the adsorbate and induce a charge transfer (CT) enhancement (2 orders of magnitude). This CT enhancement is explained by the fact that the new electronic states could potentially coincide with the energy of the incident light, leading to resonant Raman scattering through electronic excitation of the coupled molecule-surface system. ${ }^{9,19}$ Raman shifts up to $30 \mathrm{~cm}^{-1}$ are then expected. ${ }^{20}$

- Changes in local microenvironment including numerous parameters such as temperature, hydrophobicity, pH, ionic strength, buffer that are hardly mastered. They induce small Raman shifts that are responsible for the spectral broadening of Raman bands in SERS. ${ }^{21,22}$ 
- Finally, the conformation landscape of the molecules has to be considered. Computationally, the time decay of conformational structure modification is around $3 \mathrm{~ns}$; this time can be longer when the molecules are blocked onto the surface. Large spectral shifts have been reported experimentally. ${ }^{23}$

Although statistics in Raman intensity on macromolecule was largely explored, the analysis has not yet been conclusive to isolate the origin of above sources of fluctuations. Furthermore, no significant statistical study of the Raman shift has been performed to the best of our knowledge.

For investigating these spectral fluctuations, we propose to exploit the auto-correlation that was used heavily and successfully in fluorescence correlation spectroscopy (FCS) ${ }^{24}$ and Dynamic Light Scattering (DLS). ${ }^{25}$ Raman correlation spectroscopy exists but it is referred to an other method consisting in calculating the correlation matrix between Raman spectra. ${ }^{26}$ The autocorrelations of SERS kinetics have already been performed for the whole spectrum ${ }^{27}$ and for some specific Raman bands selected by narrow bandpass filters. ${ }^{28,29}$ However, due to the limited bandwidth of such filters (around $1 \mathrm{~nm}$ corresponding to a Raman shift of 15 $\mathrm{cm}^{-1}$ ) the conformational information of molecules is not accessible.

Furthermore, to the best of our knowledge, the cross-correlation analyses of these fluctuations for each isolated Raman line considered as single photon emitter have not yet been performed. We believe that the analysis of auto-correlation functions of characteristic line shifts $\left(G_{I}\right.$ and/or $\left.G_{\nu}\right)$ should be able to track kinetic changes in conformation or chemisorption in relation to environmental variation.

Our objective is to identify specific indicators allowing conformational changes in macromolecules compared to other sources of fluctuations. As macromolecule, we choose an amino acid, the tryptophan (Trp), that has been extensively studied in spontaneous Raman spectroscopy. ${ }^{23,30,31}$ All bands have been assigned previously. ${ }^{23,32,33}$ In this work, we focus on three main bands chosen explicitly because they are known to be specific markers:

- the Raman band at $930 \mathrm{~cm}^{-1}$ is attributed to both the stretching of $\mathrm{COO}^{-}$and the 
rocking of $\mathrm{NH}_{3}^{+} ; 3$ it can thus be considered as a marker of surface binding. Indeed L-tryptophan has a zwitterionic form and it has been shown that both carboxylate $(\mathrm{COOH})$ group and amino $\left(\mathrm{NH}_{2}\right)$ group of L-tryptophan are the preferred binding sites to attach to metallic surface. ${ }^{15,16}$

- a doublet with Raman band at 1340 and $1360 \mathrm{~cm}^{-1}$ corresponding to an indole ring vibration (denoted W7) serves as local hydrophobicity indicator of the solvent because the intensity ratio of this doublet $(1360 / 1340)$ increases when the microenvironment is strongly hydrophobic. ${ }^{23}$

- the Raman band around $1550 \mathrm{~cm}^{-1}$ is attributed to an other indole ring vibration (denoted W3). It has been shown that this band is affected by the torsion angle of the side chain, it is thus used as a conformation marker. ${ }^{23}$

We describe our methodology in details so that the approach may be valid in a more general case.

\section{MATERIALS and METHODS}

\section{SERS experiment}

Our SERS active substrate is based on a self-assembly of gold nanoparticles (GNPs) capped with microfluidic channels. GNPs of $80 \mathrm{~nm}$ in diameter and complex surface shape (raspberrylike) were synthesized following the protocol described in. ${ }^{34}$ Two-dimensional self-assembly is obtained spontaneously after air drying of a drop of the nanoparticles solution. Typical interparticle distance is comprised between 2 and $10 \mathrm{~nm}$ varying locally due to surface roughness. After lift-off, only the particles deposited inside the channels remain on the glass surface. The substrate is then exposed to an oxygen plasma for 30 min to remove all traces of surfactants. The substrate is finally capped with microfluidic channels of $100 \mu \mathrm{m}$ width and $50 \mu \mathrm{m}$ thickness. ${ }^{35}$ 
L-Tryptophan was purchased from Sigma-Aldrich. It was diluted in deionized water to achieve a concentration $C_{o}$ of $10^{-8} \mathrm{~mol} / \mathrm{L}$. A peristaltic pump is used to flow the amino acid through the SERS active volume. A flow rate of $2 \mu \mathrm{L} / \mathrm{min}$ was used.

Dynamic SERS measurements are conducted with use of a custom-built epi-confocal Raman microscope equipped with a $60 \times$ water immersion objective lens (1.2 NA, Nikon). SERS spectra are recorded with a spectrometer $\left(600\right.$ lines. $\mathrm{mm}^{-1}$, overall spectral resolution is $\left.2 \mathrm{~cm}^{-1}\right)$ associated with a cooled CCD camera $(1024 \times 256$ pixels) cadenced at 2 spectra per second. The magnitude of the signal is expressed in terms of count rate (cts.s $\left.{ }^{-1}\right)$. A 784 $\mathrm{nm}$ laser line is chosen to limit photoluminescence from the gold nanoparticles. Laser power is kept constant at $100 \mu \mathrm{W}$ at the objective back pupil plane.

\section{Correlation analysis}

Experimental data were analyzed using a home made program developed with MATLAB and Statistics toolbox (version R2014B, The MathWorks Inc.) based on standard algorithm for finding local maxima.

More than 150 temporal series of $600 \mathrm{~s}$ have been analyzed. From this whole data set, 6000 SERS spectra of similar enhancement (namely having signal to noise ratio larger than 100) were selected and then background corrected (by subtracting an average offset). For auto-correlation and cross-correlation analysis, only series having more than 100 consecutive spectra for the three selected Raman bands: $910-950 \mathrm{~cm}^{-1}, 1330-1370 \mathrm{~cm}^{-1}$ and $1530-1570$ $\mathrm{cm}^{-1}$ were considered. Finally we found 3 traces for Raman band at $910-950 \mathrm{~cm}^{-1}, 2$ traces for $1330-1370 \mathrm{~cm}^{-1}$ and 4 traces for $1530-1570 \mathrm{~cm}^{-1}$.

The temporal auto-correlation function $G$ is the correlation of a time series with itself shifted by a time lag $\tau$ as a function of $\tau$. The auto-correlation functions corresponding to SERS intensity $I$ and Raman shift $\nu$ are given by 


$$
\begin{aligned}
& G_{I}(\tau)=\frac{\langle\delta I(t) \delta I(t+\tau)\rangle}{\langle I(t)\rangle^{2}} \\
& \text { and } \\
& G_{\nu}(\tau)=\frac{\langle\delta \nu(t) \delta \nu(t+\tau)\rangle}{\langle\nu(t)\rangle^{2}}
\end{aligned}
$$

where $\delta I(t)=I(t)-\langle I(t)\rangle$ and $\delta \nu(t)=\nu(t)-\langle\nu(t)\rangle$ are the deviations from the mean intensity $\langle I\rangle$ and the mean Raman shift $\langle\nu\rangle$.

The cross-correlation function $G_{I \nu}$ is the correlation between the time series of intensity $I(t)$ and Raman shift $\nu(t)$ as defined as

$$
G_{I \nu}(\tau)=\frac{\langle\delta I(t) \delta \nu(t+\tau)\rangle}{\langle I(t)\rangle\langle\nu(t)\rangle}-1
$$

These correlation functions were fitted by four standard models summarized in Table 1. Convection and diffusion are the two physical mechanisms by which molecules move from the solution into the SERS active area. Because the SERS signal is mainly emitted from the surface, we limit our study to two-dimensional models. The pure 2D free diffusion governed by the standard Brownian motion probed within Gaussian laser excitation intensity profile was investigated using the auto-correlation modeled by: ${ }^{24}$

$$
G_{d}(\tau)=\frac{G(0)}{1+\frac{\tau}{\tau_{d}}}
$$

where $\tau_{D}$ is the characteristic residence time related to the diffusion coefficient $D$ and the 
beam radius $w$ of the excited volume by:

$$
\tau_{D}=\frac{w^{2}}{4 D}
$$

The pure $2 \mathrm{D}$ convection depends on many parameters : the nature and geometry of the surface, changes in temperature and the thermophysical properties of the fluid. However it can be approximated to an active transport (or translation process) whose autocorrelation function $G_{c}(\tau)$ is given by ${ }^{36}$

$$
G_{c}(\tau)=G(0) \times \exp \left(-\frac{\tau^{2}}{\tau_{c}^{2}}\right)
$$

where $\tau_{c}$ is the characteristic time of convection. The molecule convective velocity $V_{c}$ is given by:

$$
V_{c}=\frac{w}{\tau_{c}}
$$

Combination of 2D free diffusion and convection was also tested using the function $G_{d c}(\tau):{ }^{36}$

$$
G_{d c}(\tau)=\frac{G(0)}{1+\frac{\tau}{\tau_{D}}} \times \exp \left(-\frac{\tau^{2}}{\tau_{c}^{2}} \times \frac{1}{1+\frac{\tau}{\tau_{D}}}\right)
$$

From this model, we can estimate the nondimensional Péclet number $P e$ which represents the ratio of transport by convection to the perpendicular diffusion:

$$
P e=\frac{\tau_{c}}{\tau_{D}}
$$

Finally, the potential adsorption of molecule onto the surface may induce at the chemical vibration level some unimolecular isomerization-like processes between two states A and B such as $A \underset{k_{A B}}{\stackrel{k_{B A}}{\rightleftharpoons}} B$ where $k_{A B}$ and $k_{B A}$ are the rate constants of the state changes between $\mathrm{A}$ and $\mathrm{B}$. The kinetic chemical equilibrium was then investigated through the auto-correlation 
function $G_{s}(\tau)^{37}$

$$
G_{s}(\tau)=G(0) \times\left(1-K+K \exp \left(-\frac{\tau}{\tau_{s}}\right)\right)
$$

where $K$ is the fraction of molecule in the B state and it is defined by :

$$
K=\frac{k_{B A}}{k_{A B}+k_{B A}}
$$

and $\tau_{s}$ is the lifetime of the changes in states $\mathrm{A}$ and $\mathrm{B}$, which is equal to: $\tau_{s}=\left(k_{A B}+k_{B A}\right)^{-1}$.

Table 1: Models of auto-correlation analysis: $D$ is the diffusion coefficient, $\omega$ the beam radius, $V_{c}$ the convection velocity, $k_{A B}$ and $k_{B A}$ are the equilibrium constants between $\mathrm{A}$ and $\mathrm{B}, P e$ is the Péclet number.

\begin{tabular}{|c|c|c|}
\hline Model & Equation & Outup Parameters \\
\hline 2D diffusion & $G_{d}(\tau)=G(0) \frac{1}{\left(1+\tau / \tau_{D}\right)}$ & $D=\omega^{2} /\left(4 \tau_{D}\right)$ \\
\hline Convection & $G_{c}(\tau)=G(0) \exp \left[-\left(\tau / \tau_{c}\right)^{2}\right]$ & $V_{c}=\omega / \tau_{c}$ \\
\hline Diffusion - Convection & $G_{d c}(\tau)=\frac{G(0)}{\left(1+\tau / \tau_{D}\right)} \exp \left[-\left(\tau / \tau_{C}\right)^{2} \frac{1}{1+\tau / \tau_{D}}\right]$ & $P e, D, V_{c}$ \\
\hline Chemical states & $G_{s}(\tau)=G(0)\left(1-K+K \exp \left[-\frac{\tau}{\tau_{s}}\right]\right)$ & $\begin{array}{c}k_{B A}=K / \tau_{s} \\
k_{A B}=(1-K) / \tau_{s}\end{array}$ \\
\hline
\end{tabular}

\section{RESULTS and DISCUSSION}

Representative series are shown in Figure 1. Chromatograms enabling comparison between the three Raman bands of $40 \mathrm{~cm}^{-1}$ width as defined in the introduction are indicated in Figure 1-A1, B1 and C1. The evolution of the intensity $I(\nu, t)$ is followed during more than $60 \mathrm{~s}$ for each Raman band using a color gradient. The position of the peak apex is then detected and shown in red line; the intensity of the corresponding peak is followed in Figure 1-A3, B3 and C3. The spectrum corresponding to the cumulative average intensity is represented on the right-hand side.

Diffusion and convection processes of the Raman lines can be visually inspected. In the single molecule regime, systematic deviations of dynamics from Brownian motion have already been observed with intrinsic variability. ${ }^{38-40}$ The diffusion is observed by displace- 

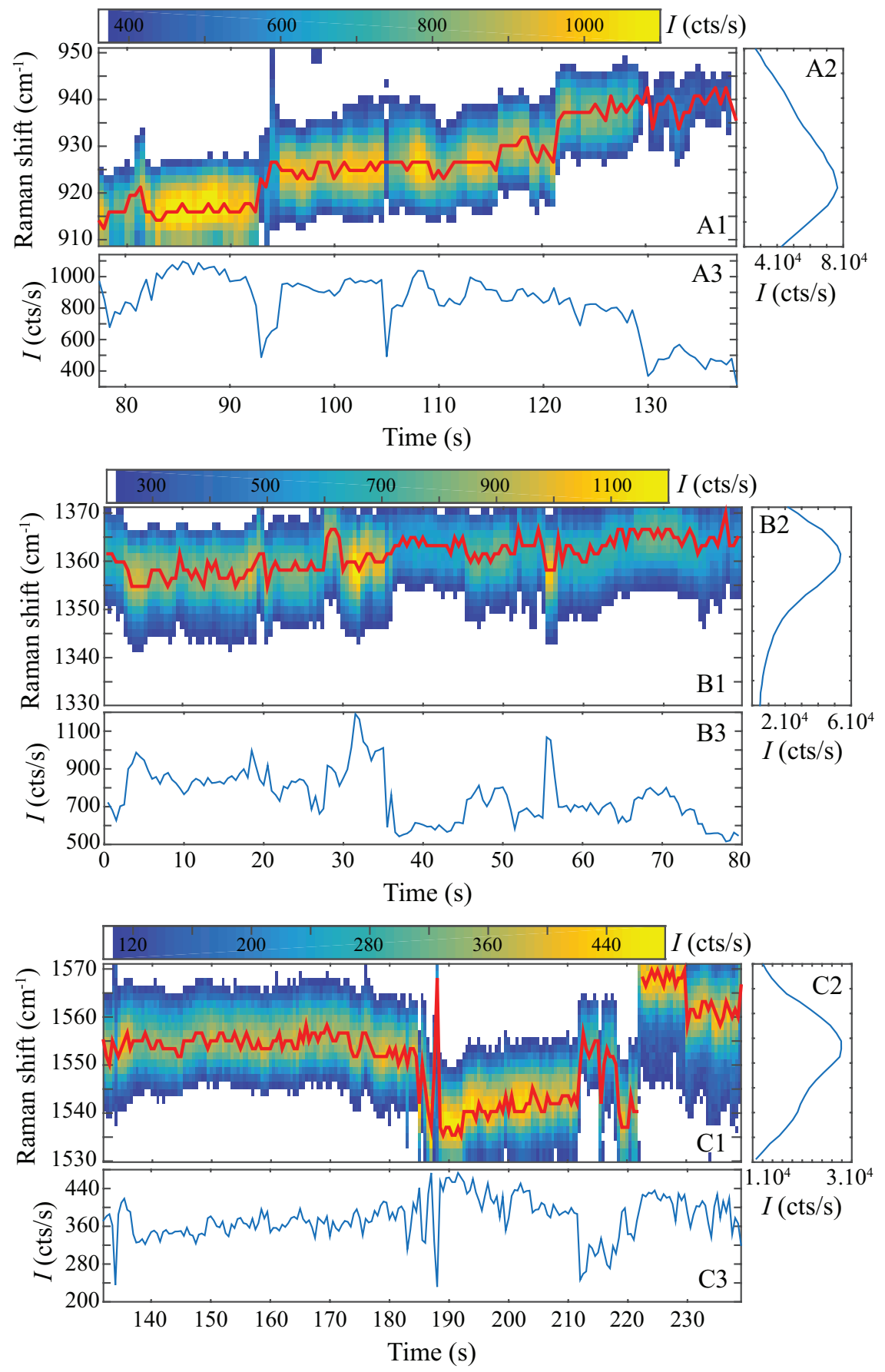

Figure 1: Surface enhanced Raman chromatograms of three specific Raman lines (A1: 910$950 \mathrm{~cm}^{-1}$, B1: $1330-1370 \mathrm{~cm}^{-1}, \mathrm{C} 1: 1530-1570 \mathrm{~cm}^{-1}$ ) of Trp in the single molecule regime. The intensity is shown using a color gradient. Wavenumber of the most intense Raman bands are plotted in red line.The cumulative SERS spectra for each Raman band are plotted respectively in A2, B2 and $\mathrm{C} 2$. Intensity of the most intense Raman bands are followed with time in A3, B3 and C3. Spectra were acquired every $0.5 \mathrm{~s}$. 
ments rather chaotic whose microscopic origin is responsible for the broadening of Raman peaks. Convective motions are characterized by larger and more abrupt displacements, such as jumps that not only participate in broadening, but also shoulder in the spectrum.

Qualitatively on the Raman shift displacements (red curve), the diffusive character is more pronounced on the $1340 \mathrm{~cm}^{-1}$ band (Fig. 1-B1) with a symmetrical Raman band (Fig. 1-B2) around a single frequency at $1360 \mathrm{~cm}^{-1}$ without shoulder. The peak at $1340 \mathrm{~cm}^{-1}$ is not observed here, which means that Trp is in a largely stable and nonpolar neighborhood, such as the vicinity of a gold surface. It is worth noting that this band exhibits the largest fluctuations in intensity (Fig. 1-B3), but not in wave number.

The convective feature with characteristic jumps is found for the two other bands centered at 930 and $1550 \mathrm{~cm}^{-1}$, thereby leading to a skewness in the spectral profile (Fig. 1-A2-C2). In Fig. 1-C1, at least 3 energy levels are observed in Raman Shift, typically $1540 \mathrm{~cm}^{-1}, 1550$ $\mathrm{cm}^{-1}$, and $1570 \mathrm{~cm}^{-1}$. This jumps reflects changes in the torsion angle of the side chain, typically between $60^{\circ}$ and $120^{\circ} .{ }^{23}$ Changing the molecular conformations does not entail inevitably the swing of intensity (Fig. 1-A3).

Fig. 1-A1 typically shows a step-wise process between various energy levels at $915 \mathrm{~cm}^{-1}$, $925 \mathrm{~cm}^{-1}$ and $930 \mathrm{~cm}^{-1}$. The first stage centered on $915 \mathrm{~cm}^{-1}$ is very intense, very fluctuating in intensity over $20 \mathrm{~cm}^{-1}$; then further stages are abruptly refined, with a narrow Raman line less intense but more stable. Several hypotheses can be drawn to explain the peak locations. First the $930 \mathrm{~cm}^{-1}$ Raman band is assigned to several vibrations, namely the stretching of $\mathrm{COO}^{-}$, the rocking of $\mathrm{NH}_{3}^{+}$and $\mathrm{CH}_{2}$ and the twisting of $\mathrm{H}$ in benzene ring. ${ }^{33}$ The most likely explanation of the multistep process seems to come from a competitive adsorption of Trp on the gold surface. Thus the first step would be a $\mathrm{COOH}$ and $\mathrm{NH}_{2}$ coadsorption competition in favor of a predominant form of $\mathrm{COO}^{-}$. Indeed, it was found theoretically that the combination of multiple bounds (6 Au-indole $+1 \mathrm{Au}$-COOH group, $-2.6 \mathrm{eV}))$ is more favorable and stronger compared to the complex of 3 bonds with $\mathrm{NH}_{2}(-2$ eV). ${ }^{41}$ Both carboxyl and amino groups are known to develop charge transfer with metallic 
surface (gold, graphene). Whatever the assignment of the vibration to the amino and/or the acid group, the Raman shifts leading to an inhomogeneous broadening over $30 \mathrm{~cm}^{-1}$ and the increase in intensity in the blue shift bring altogether the signature characteristics of an electronic transfer of the metal to the adsorbate. ${ }^{20}$

The intensity fluctuations (Fig. 1.A3-B3-C3) behave clearly in a dual diffusive and convective manner for all the bands concerned, which makes a proper interpretation much more difficult.

In order to quantify kinetic parameters, the characterization of the data ordering is of primary interest in laying down the data structure. Figure 2 shows the auto-correlation fonctions $G_{I}(\tau)$ and $G_{\nu}(\tau)$ corresponding to the internal correlations within a time series of the Raman lines at $930 \mathrm{~cm}^{-1}$. The initial value $G(0)$ is equal to the variance of the time series divided by its mean square and it corresponds to the maximal correlation. When the time lag increases, the correlation is less pronounced and it tends to 0 .

Table 2: Output parameters obtained after fitting intensity and wavenumber auto-correlations of the $930 \mathrm{~cm}^{-1}$ Raman band with models of diffusion, convection , mixed diffusion-convection and chemical advection.

\begin{tabular}{|l|ccc|ccc|}
\hline & \multicolumn{3}{|c|}{ Intensity } & \multicolumn{3}{c|}{ Wavenumber } \\
\hline models & Fit & Output & res & Fit & Output & res \\
\hline diff. & $\tau_{D}=4.7 \mathrm{~s}$ & $\mathrm{D}=4.10^{-15} \mathrm{~m}^{2} \mathrm{~s}^{-1}$ & $5.10^{-4}$ & $\tau_{D}=5.9 \mathrm{~s}$ & $\mathrm{D}=3.10^{-15} \mathrm{~m}^{2} \mathrm{~s}^{-1}$ & $6.10^{-3}$ \\
\hline conv. & $\tau_{C}=9.7 \mathrm{~s}$ & $V_{C}=27 n m . s^{-1}$ & $2.10^{-3}$ & $\tau_{c}=12.9 \mathrm{~s}$ & $V_{C}=20{\mathrm{~nm} . \mathrm{s}^{-1}}$ & $10^{-2}$ \\
\hline diff-conv & $\tau_{D}=5.9 \mathrm{~s}$ & $\mathrm{Pe}=3.2$ & $3.10^{-4}$ & $\tau_{D}=10.4 \mathrm{~s}$ & $\mathrm{Pe}=1.6$ & $3.10^{-3}$ \\
& $\tau_{c}=19.1 \mathrm{~s}$ & & & $\tau_{c}=16.8 \mathrm{~s}$ & & \\
\hline chemical & $\mathrm{K}=0,96$ & $k_{B A}=0.1 \mathrm{~s}^{-1}$ & $4.10^{-4}$ & $\mathrm{~K}=1.0$ & $k_{B A}=0.08 s^{-1}$ & $3.10^{-3}$ \\
& $\tau_{s}=8.6 \mathrm{~s}$ & $k_{A B}=4.10^{-3} \mathrm{~s}^{-1}$ & & $\tau_{s}=12.6 \mathrm{~s}$ & $k_{A B}=0 \mathrm{~s}^{-1}$ & \\
\hline
\end{tabular}

The auto-correlation defines what is called the integral scale, namely the time during which the signal can remain deterministic. The auto-correlation at lag zero is the variance of the time series divided by its mean square; at $\tau=0.5 \mathrm{~s}, G_{I}(\tau)$ and $G_{\nu}(\tau)$ indicate a weak correlation $G_{I}(0.5)=0.06$ and $G_{\nu}(0.5)=0.15$, respectively; the strength of the correlation dies down as $\tau$ increases up to $20 \mathrm{~s}$. The life-time of a correlation $\tau_{a c f}$ defined when the 

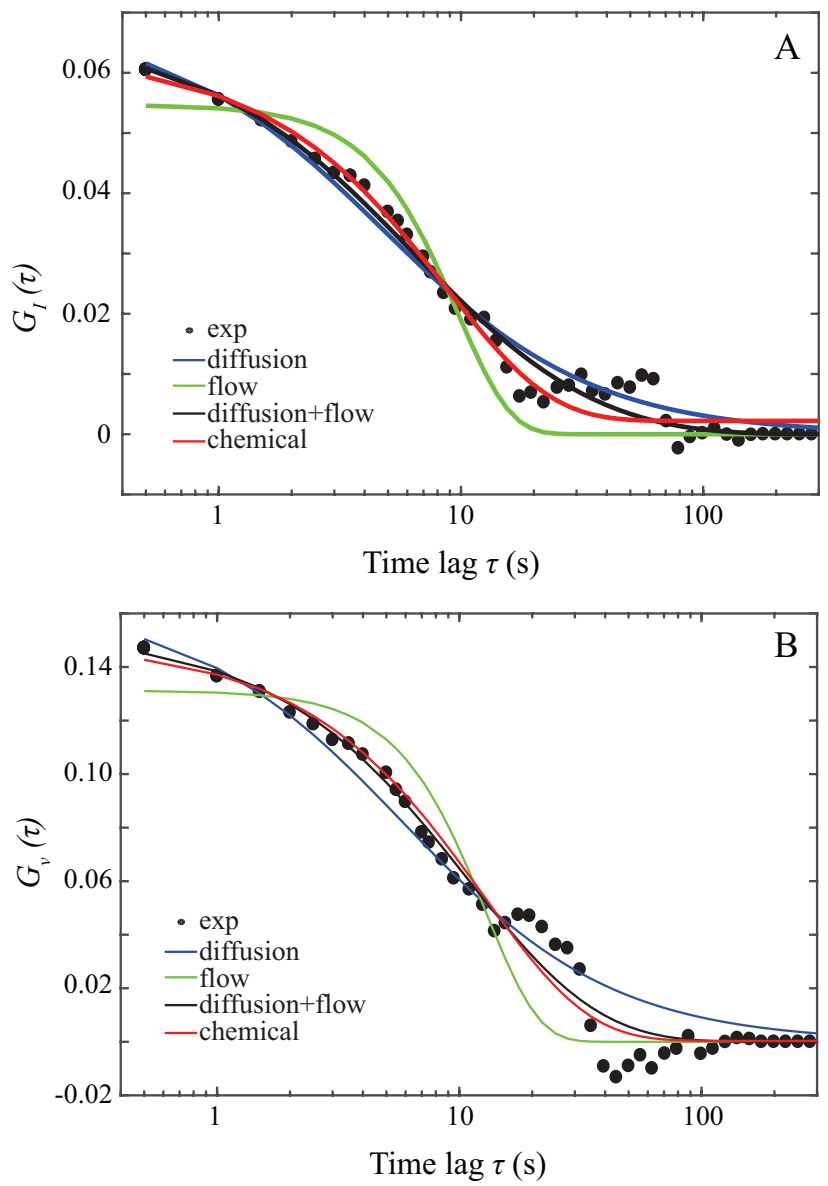

Figure 2: Auto-correlation analysis of amino-acid at the $930 \mathrm{~cm}^{-1}$ Raman line (A) Intensity auto-correlation. (B) Raman shift auto-correlation. Experimental auto-correlation are plotted in black dots and have been fitted with several models: diffusion (blue line), convection (green line), mixed diffusion and convection (black line), chemical advection (red line). 
correlation is larger than $\frac{G(0)}{2}$ is the same for the signal $I$ and $\nu: \tau_{a c f}=10 \mathrm{~s}$. This relatively long time is a first indication of the trapping of the molecule by the surface. The decay rate of $G$ confirms that the correlation can be explained by standard models in surface physics. After fitting with previously described models (cf. Table 2), it appears that diffusion and convection models taken separately do not account for this correlation. Nevertheless, the combined diffusion-convection model and the chemical advection model both fit better the experimental points. The fitting times and physical outputs are collected in Table 2 .

The diffusion coefficient $D$ is deduced from $\tau_{D}$ and the laser beam radius $w$ (cf. Equ. 5) which is estimated about $260 \mathrm{~nm}$ in our experiment $(1.2 \mathrm{NA}, \lambda=784 \mathrm{~nm}) . D$ is the same order of magnitude for both I and $\nu$ and do not exceed $4.10^{-15} \mathrm{~m}^{2} \mathrm{~s}^{-1}$. The diffusion coefficient of a free molecule $D_{o}$ in water is approximately $10^{-10} \mathrm{~m}^{2} \cdot \mathrm{s}^{-1} \cdot{ }^{42}$ This limited diffusion is explained by the surface energy $E_{s}=-k_{B} T \cdot \ln \left(\frac{D}{D_{o}}\right)$ around $10 \times$ the thermal energy $k_{B} T$, which is larger than the Van der Waals energy and in better agreement to an electrostatic or ionic interaction between the molecule and the surface. ${ }^{43}$

Concerning the convection, we can deduce a mean convective velocity $V_{C}$ (Equ. 7) by using the same beam radius $\omega, V_{C}<30 \mathrm{~nm} \cdot \mathrm{s}^{-1}$ (Table 2) seems to be very slow. The product $V_{C} \cdot \omega=8.10^{-15} \mathrm{~m}^{2} \cdot \mathrm{s}^{-1}$ does not agree with expected order of magnitude of the thermal diffusivity $\left(\kappa=1 \cdot 10^{-7} \mathrm{~m}^{2} \cdot \mathrm{s}^{-1}\right)$ and kinematic viscosity $\left(\mu_{k}=10^{-6} \mathrm{~m}^{2} \cdot \mathrm{s}^{-1}\right) \cdot{ }^{44}$ The Marangoni effect is the mass transfer at the surface due to a gradient of the surface tension which can be caused by both a temperature gradient but also by a concentration gradient. Although the expected localized concentration gradient can be highly pronounced at the nanoscale due to the nonhomogeniety of surface and the high enhancement of electrical field due to the plasmonic effect, the Marangoni effect does not explain the slow process of these experiences.

The process is better described by a mixed convection-diffusion process. A Péclet number (Equ. 9) close to 1 indicates typically an intermediary system where the convection and the diffusion carry the same weight. Due to the presence of the surface, the diffusion is 
limited compared to the brownian motion. In addition, since the molecule is adsorbed on the surface, a convective process occurs much lower than microfluidics device. Both physical mass transports contribute then to a mixing, similar to a turbulent flow. Such intermediary regime which was not observed in FCS (diffusive) or engineering microfluidics (convective) appears to be a common feature of surface enhanced processes, which fosters the emergence of multistep processes and fastens surface reaction such as chemical-like sorption.

In fact, a chemical two states model also provides a good description of the kinetics of the Raman line at $930 \mathrm{~cm}^{-1}$. The fitted parameter $K$ (cf. Table 2 ) is close to 1 for both $I$ and $\nu$, which means a directional and non-reversible exchange rates from Raman state A to state $\mathrm{B}\left(k_{A B}<<k_{B A}\right)$. The simple two states model is therefore well-adapted to explain the dynamics of Raman fluctuations. Furthermore, since $K \simeq 1$, the equation 10 simplifies to :

$$
G_{s}(\tau)=G(0) K \exp \left(-\frac{\tau}{\tau_{s}}\right)
$$

where $\tau_{s}$ can be then obtained directly from the the half-time in the auto-correlation figure. From this half time $\tau_{s, I} \simeq 10 s$ and the fraction $K=0.96$, we found that $k_{B A}=K / \tau_{s}=0.1 s^{-1}$ and $k_{A B}=(1-K) / \tau_{s}=4 \cdot 10^{-3} s^{-1}$.

The Figure 3 shows for comparison the cross-correlations $G_{I}$ and $G_{\nu}$ for the two others lines at 1340 and $1550 \mathrm{~cm}^{-1}$. $G_{I}$ is lower for the lines that do not correspond to a vibration related to an adsorption site. However $G_{\nu}$ reflects the complex nature of the line 1550 $\mathrm{cm}^{-1}$ with two processes in the Raman shift (Fig. $3 \mathrm{~B}$ ). At short time, $G_{\nu}$ is governed by a chemical law similar to the $930 \mathrm{~cm}^{-1}$. At longer lag time around $\tau=40 \mathrm{~s}, G_{\nu}$ shows negative auto-correlation changes. This anticorrelation is usually explained by an alternating pattern or a two-step process. This negative bell shape seems to be an indicator of the low conformational changes which we have attributed previously to the $1550 \mathrm{~cm}^{-1}$.

Results of the best fitting models are displayed in Table 3 and Table 4. 

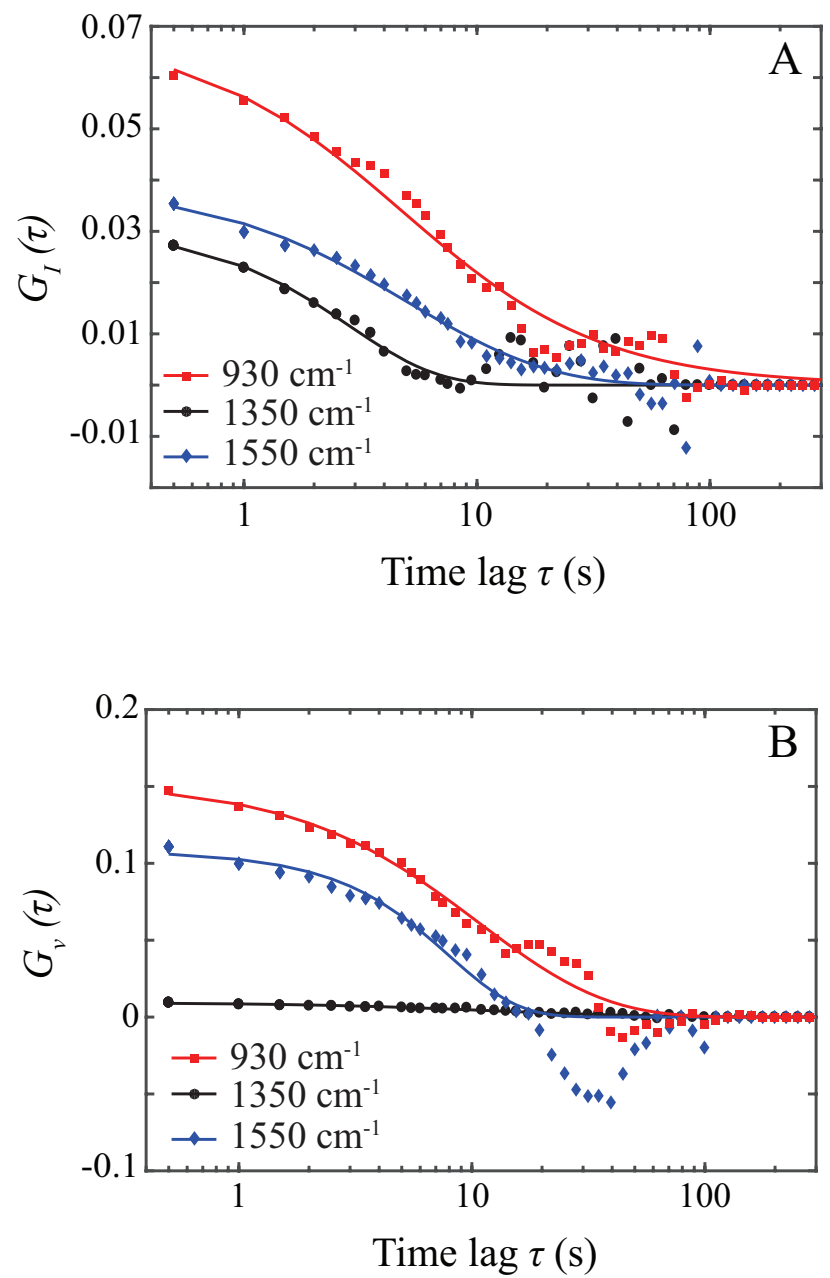

Figure 3: Auto-correlations analysis of Trp amino-acid. (A) Auto-correlation of SERS intensity. (B) Auto-correlation of spectral fluctuations. Three Raman bands are considered: 910-950 $\mathrm{cm}^{-1}$ (red squares), 1330-1370 $\mathrm{cm}^{-1}$ (black dots) and $1530-1570 \mathrm{~cm}^{-1}$ (blue diamonds). Experimental points are fitted with the model combining free diffusion and active transport (indicated in plain line).

Table 3: Results (95\% confidence bounds) of auto-correlation fitted with diffusion-convection model for the three Raman lines.

\begin{tabular}{|c|c|c|c|c|}
\hline Raman line & & $\tau_{d}(\mathrm{~s})$ & $\tau_{c}(\mathrm{~s})$ & $\mathrm{Pe}=\frac{\tau_{c}}{\tau_{d}}$ \\
\hline $930 \mathrm{~cm}^{-1}$ & $\mathrm{I}$ & {$[5.0-6.9]$} & {$[14.2-24.0]$} & 3.2 \\
& $\nu$ & {$[8.0-12.8]$} & {$[14.3-19.3]$} & 1.6 \\
\hline $1340 \mathrm{~cm}^{-1}$ & $\mathrm{I}$ & {$[0-9.3]$} & {$[2.5-3.8]$} & 0.8 \\
& $\nu$ & None & None & None \\
\hline $1550 \mathrm{~cm}^{-1}$ & $\mathrm{I}$ & {$[3.1-6.2]$} & {$[7.0-11.9]$} & 2.0 \\
& $\nu$ & {$[0-62]$} & {$[6.4-10.0]$} & 0.4 \\
\hline
\end{tabular}


Table 4: Results (95\% confidence bounds) of auto-correlation fitted with chemical model for the three Raman lines.

\begin{tabular}{|c|c|c|c|}
\hline Raman line & & $K$ & $\tau_{s}(\mathrm{~s})$ \\
\hline $930 \mathrm{~cm}^{-1}$ & $\mathrm{I}$ & {$[0.95-0.98]$} & {$[7.8-9.4]$} \\
& $\nu$ & {$[0.98-1.0]$} & {$[11.3-13.8]$} \\
\hline $1340 \mathrm{~cm}^{-1}$ & $\mathrm{I}$ & {$[0.93-1.0]$} & {$[1.8-3.0]$} \\
& $\nu$ & None & None \\
\hline $1550 \mathrm{~cm}^{-1}$ & $\mathrm{I}$ & {$[0.97-1.0]$} & {$[5.4-7.2]$} \\
& $\nu$ & {$[0.94-1.0]$} & {$[4.7-8.9]$} \\
\hline
\end{tabular}

The differences between the Péclet number Pe shows that each Raman line behaves distinctly whether in intensity or in Raman shift. Typically, the $930 \mathrm{~cm}^{-1}$ line characteristic of adsorption, is rather dominated by a forced convective movement ( $\mathrm{Pe}>1.6)$. On the other hand, the diffusion $(\mathrm{Pe}<0.5)$ associated with a large range of $\tau_{d}$ prevails for the spectral fluctuations of the $1550 \mathrm{~cm}^{-1}$ line that we attributed to the conformation marker. Moreover, a diffusive behavior in wave number does not prohibit convective shifts in intensity for this band. The Pe number seems to be a good indicator to discern between the vibration modes forced by the surface and the rather free undamped vibration modes.

The application of the chemical model to all lines (Table 4) confirms the two iso-states with $K=1$. The correlations appear longer for the Raman shift than for the intensity. The fastest correlation times $(\tau=2 \mathrm{~s})$ are found in intensity for the environmental marker line. The modes forced by the surface are logically the slowest $(\tau=13 \mathrm{~s})$, while the conformational mode $\tau=8 \mathrm{~s}$ ) are reduced compared to the surface mode.

For investigating more deeply the potential relation between spectral and intensity fluctuations, we have applied the cross-correlation $G_{I \nu}(\tau)$ in Fig. 4. Cross-correlation can be first considered as negligible for two Raman bands: the inactive band at $1340 \mathrm{~cm}^{-1}$ and the conformation band at $1550 \mathrm{~cm}^{-1}$. This confirms the fact that the spectral variations (which are related to the orientation of the side chain) does not necessarily induce changes 


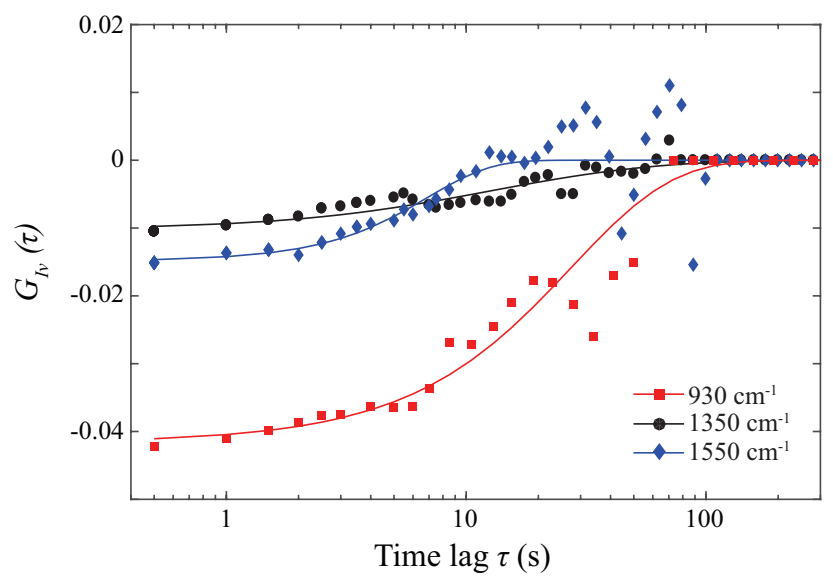

Figure 4: Cross-correlation analysis between SERS intensity and spectral fluctuations for single amino-acid regime. Three Raman bands are considered: $910-950 \mathrm{~cm}^{-1}$ (red squares), 1330-1370 $\mathrm{cm}^{-1}$ (black dots) and $1530-1570 \mathrm{~cm}^{-1}$ (blue diamonds). Experimental points are fitted with the chemical model (indicated in plain line).

in SERS intensity. The largest negative cross-correlation is obtained for the $930 \mathrm{~cm}^{-1} \mathrm{Ra}-$ man band. Note that $G_{I \nu}(0)$ is negative indicating that SERS intensity and Raman shift are anti-correlated. In other words, an increase in $I$ is strongly related to a decrease in Raman shift $\nu$. This phenomenon can be easily explained by a charge transfer between the surface and the amino-acid as already reported in. ${ }^{20}$ During the adsorption, a charge transfer occurs from the surface to the molecule, thereby increasing the energy levels and thus decreasing the Raman shift. This charge transfer will also chemically enhanced the Raman signal, thereby inducing an increase of the SERS intensity. When the interaction disappears, this chemical enhancement is vanished and the Raman shift increases reversibly. The fact that $G_{I \nu}(\tau)$ follows the same dynamics of diffusion limited chemical reaction described above for the auto-correlation confirms that the charge transfer induced enhancement is of chemical origin.

Previous models can also be used to fit the cross-correlation signal (Table 5). The crosscorrelation thus shows that when the charge transfer exists $\left(930 \mathrm{~cm}^{-1}\right)$, the memory of the signals is persistent over long duration up to forty seconds, whereas this life time remains short for the conformational line $\left(1550 \mathrm{~cm}^{-1}\right)$. 
Table 5: Results (95\% confidence bounds) of cross-correlation fitted with chemical model for the three Raman lines.

\begin{tabular}{|c|c|c|}
\hline Raman line & $K$ & $\tau_{s}(\mathrm{~s})$ \\
\hline $930 \mathrm{~cm}^{-1}$ & {$[0.90-1.0]$} & {$[17.3-38.6]$} \\
\hline $1340 \mathrm{~cm}^{-1}$ & {$[0.94-1.0]$} & {$[15.1-24.3]$} \\
\hline $1550 \mathrm{~cm}^{-1}$ & {$[0.91-1.0]$} & {$[3.1-8.8]$} \\
\hline
\end{tabular}

\section{CONCLUSION}

This study confirms that the spectral variations are not due to an electromagetic effect (modification of the enhanced field) but originate from both a charge transfer between the molecule and the surface, as intuited by others ${ }^{9}$ and the conformations changes. We have investigated the interest of simple correlation functions in discerning between the chemical effect and the conformations changes. Indicator such as the Péclet number Pe was used to discern the convective and diffusive mass transport.

The negative cross-correlation between the intensity and the Raman shift clearly demonstrates a slow charge transfer at $930 \mathrm{~cm}^{-1}$ band which follows a forced surface reaction $\left(\mathrm{Pe}>1\right.$ for $G_{I}$ and $G_{\nu}$ ) governed by a competition between the carboxyl and amino groups both having an affinity to the gold surface.

The negative auto-correlation of Raman shift is an indicator of faster alternating pattern such as observed at $1550 \mathrm{~cm}^{-1}$ and the changes in molecular conformation are governed by a diffusion limited process $(\mathrm{Pe}<1)$, such as the torsion angle of the side chain.

\section{Acknowledgement}

This work has been supported by the EIPHI Graduate School (contract "ANR-17-EURE0002"), the Région Bourgogne Franche Comté (PARI II NANO2BIO and contract CIME) and the European Union (FEDER-FSE Bourgogne 2014-2020). J.-E. C. was partly funded by the Région Bourgogne Franche Comté. 


\section{References}

(1) Lakowicsz, J. R. Principles of Fluorescence Spectroscopy, 2nd ed.; Plenum Publishers: New York, 1999.

(2) Jares-Erijman, E. A.; Jovin, T. M. FRET Imaging. Nat. Biotechnol. 2003, 21, 1387-95, 11.

(3) Micsonai, A.; Wien, F.; Kernya, L.; Lee, Y.-H.; Goto, Y.; Réfrégiers, M.; Kardos, J. Accurate Secondary Structure Prediction and Fold Recognition for Circular Dichroism Spectroscopy. Proc. Natl. Acad. Sci. U. S. A. 2015, 112, E3095-3103.

(4) Singhal, K.; Kalkan, A. K. Surface-Enhanced Raman Scattering Captures Conformational Changes of Single Photoactive Yellow Protein Molecules under Photoexcitation. J. Am. Chem. Soc. 2010, 132, 429-431.

(5) Brulé, T.; Bouhelier, A.; Yockell-Lelièvre, H.; Clément, J.-E.; Leray, A.; Dereux, A.; Finot, E. Statistical and Fourier Analysis for In-line Concentration Sensitivity in Single Molecule Dynamic-SERS. ACS Photonics 2015, 2, 1266-1271.

(6) Leray, A.; Brulé, T.; Buret, M.; Colas des Francs, G.; Bouhelier, A.; Dereux, A.; Finot, E. Sorting of Single Biomolecules based on Fourier Polar Representation of Surface Enhanced Raman Spectra. Sci. Rep. 2016, 6, 20383.

(7) Nie, S.; Emory, S. R. Probing Single Molecules and Single Nanoparticles by SurfaceEnhanced Raman Scattering. Science 1997, 275, 1102-6, 5303.

(8) Xu, H.; Bjerneld, E. J.; Käll, M.; Börjesson, L. Spectroscopy of Single Hemoglobin Molecules by Surface Enhanced Raman Scattering. Phys. Rev. Lett. 1999, 83, 43574360.

(9) Weiss, A.; Haran, G. Time-Dependent Single-Molecule Raman Scattering as a Probe of Surface Dynamics. J. Phys. Chem. B 2001, 105, 12348-12354. 
(10) Otto, A. What is observed in single molecule SERS, and why? J. Raman Spectrosc. 2002, 33, 593-598.

(11) Habuchi, S.; Cotlet, M.; Gronheid, R.; Dirix, G.; Michiels, J.; Vanderleyden, J.; De Schryver, F. C.; Hofkens, J. Single-Molecule Surface Enhanced Resonance Raman Spectroscopy of the Enhanced Green Fluorescent Protein. J. Am. Chem. Soc. 2003, $125,8446-8447$.

(12) Maruyama, Y.; Ishikawa, M.; Futamata, M. Thermal Activation of Blinking in SERS Signal. J. Phys. Chem. B 2004, 108, 673-678.

(13) Emory, S. R.; Jensen, R. A.; Wenda, T.; Han, M.; Nie, S. Re-examining the origins of spectral blinking in single-molecule and single-nanoparticleSERS. Faraday Discuss. 2006, 132, 249-259.

(14) Le Ru, E. C.; Etchegoin, P. G.; Meyer, M. Enhancement Factor Distribution around a Single Surface-Enhanced Raman Scattering Hot Spot and Its Relation to Single Molecule Detection. J. Chem. Phys. 2006, 125, 204701.

(15) Bjerneld, E. J.; Johansson, P.; Käll, M. Single Molecule Vibrational Fine-Structure of Tyrosine Adsorbed on Ag Nano-Crystals. Single Mol. 2000, 1, 239-248.

(16) Aliaga, A. E.; Osorio-Roman, I.; Garrido, C.; Leyton, P.; Cárcamo, J.; Clavijo, E.; Gómez-Jeria, J. S.; F., G. D.; Campos-Vallette, M. M. Surface enhanced Raman scattering study of L-lysine. Vib. Spectrosc. 2009, 50, 131-135.

(17) Nawrocki, G.; Cieplak, M. Aqueous Amino Acids and Proteins Near the Surface of Gold in Hydrophilic and Hydrophobic Force Fields. J. Phys. Chem. C 2014, 118, 1292912943.

(18) Clément, J.-E.; Leray, A.; Bouhelier, A.; Finot, E. Spectral Pointillism of Enhanced 
Raman Scattering for Accessing Structural and Conformational Information on Single Protein. Phys. Chem. Chem. Phys. 2016, 19, 458-466.

(19) Bizzarri, A. R.; Cannistraro, S. Lévy Statistics of Vibrational Mode Fluctuations of Single Molecules from Surface-Enhanced Raman Scattering. Phys. Rev. Lett. 2005, 94, 068303.

(20) Wright, A. M.; Howard, A. A.; Howard, J. C.; Tschumper, G. S.; Hammer, N. I. Charge Transfer and Blue Shifting of Vibrational Frequencies in a Hydrogen Bond Acceptor. J. Phys. Chem. A 2013, 117, 5435-5446.

(21) Sonntag, M. D.; Klingsporn, J. M.; Garibay, L. K.; Roberts, J. M.; Dieringer, J. A.; Seideman, T.; Scheidt, K. A.; Jensen, L.; Schatz, G. C.; Van Duyne, R. P. SingleMolecule Tip-Enhanced Raman Spectroscopy. J. Phys. Chem. C 2012, 116, 478-483.

(22) Etchegoin, P. G.; Le Ru, E. C. Resolving Single Molecules in Surface-Enhanced Raman Scattering within the Inhomogeneous Broadening of Raman Peaks. Anal. Chem. 2010, 82, 2888-2892.

(23) Takeuchi, H. Raman structural markers of tryptophan and histidine side chains in proteins. Biopolymers 2003, 72, 305-317.

(24) Elson, E. L.; Magde, D. Fluorescence Correlation Spectroscopy. I. Conceptual Basis and Theory. Biopolymers 1974, 13, 1-27.

(25) Berne, B.; Pecora, R. Dynamic Light Scattering; John Wiley: New York, 1976.

(26) Noda, I.; Marcott, C. Two-Dimensional Raman (2D Raman) Correlation Spectroscopy Study of Non-Oxidative Photodegradation of $\beta$-Carotene ${ }^{\dagger}$. J. Phys. Chem. A 2002, $106,3371-3376$.

(27) Eggeling, C.; Schaffer, J.; Seidel, C. A. M.; Korte, J.; Brehm, G.; Schneider, S.; Schrof, W. Homogeneity, Transport, and Signal Properties of Single Ag Particles Stud- 
ied by Single-Molecule Surface-Enhanced Resonance Raman Scattering. J. Phys. Chem. A 2001, 105, 3673-3679.

(28) Laurence, T. A.; Braun, G.; Talley, C.; Schwartzberg, A.; Moskovits, M.; Reich, N.; Huser, T. Rapid, Solution-Based Characterization of Optimized SERS Nanoparticle Substrates. J. Am. Chem. Soc. 2009, 131, 162-169.

(29) Laurence, T. A.; Braun, G. B.; Reich, N. O.; Moskovits, M. Robust SERS Enhancement Factor Statistics Using Rotational Correlation Spectroscopy. Nano Lett. 2012, 12, 2912-2917.

(30) Takeuchi, H.; Harada, I. Normal Coordinate Analysis of the Indole Ring. Spectrochim. Acta, Part A 1986, 42, 1069-1078.

(31) Miura, T.; Takeuchi, H.; Harada, I. Tryptophan Raman Bands Sensitive to Hydrogen Bonding and Side-Chain Conformation. J. Raman Spectrosc. 1989, 20, 667-671.

(32) Cao, X.; Fischer, G. Infrared Spectral, Structural, and Conformational Studies of Zwitterionic l-Tryptophan. J. Phys. Chem. A 1999, 103, 9995-10003.

(33) Chuang, C.-H.; Chen, Y.-T. Raman Scattering of L-Tryptophan Enhanced by Surface Plasmon of Silver Nanoparticles: Vibrational Assignment and Structural Determination. J. Raman Spectrosc. 2009, 40, 150-156.

(34) Xie, J.; Zhang, Q.; Lee, J. Y.; Wang, D. I. C. The Synthesis of SERS-Active Gold Nanoflower Tags for in Vivo Applications. ACS nano 2008, 2, 2473-2480.

(35) Brulé, T.; Yockell-Lelièvre, H.; Bouhelier, A.; Margueritat, J.; Markey, L.; Leray, A.; Dereux, A.; Finot, E. Sorting of Enhanced Reference Raman Spectra of a Single Amino Acid Molecule. J. Phys. Chem. C 2014, 118, 17975-17982.

(36) Magde, D.; Webb, W. W.; Elson, E. L. Fluorescence Correlation Spectroscopy. III. Uniform Translation and Laminar Flow. Biopolymers 1978, 17, 361-376. 
(37) Magde, D.; Elson, E. L.; Webb, W. W. Fluorescence Correlation Spectroscopy. II. An Experimental Realization. Biopolymers 1974, 13, 29-61.

(38) Metzler, R.; Jeon, J.-H.; Cherstvy, A. G.; Barkai, E. Anomalous Diffusion Models and Their Properties: Non-Stationarity, Non-Ergodicity, and Ageing at the Centenary of Single Particle Tracking. Phys. Chem. Chem. Phys. 2014, 16, 24128-24164.

(39) Bel, G.; Barkai, E. Weak Ergodicity Breaking in the Continuous-Time Random Walk. Phys. Rev. Lett. 2005, 94, 240602.

(40) Geisel, T.; Thomae, S. Anomalous Diffusion in Intermittent Chaotic Systems. Phys. Rev. Lett. 1984, 52, 1936-1939.

(41) Joshi, P.; Shewale, V.; Pandey, R.; Shanker, V.; Hussain, S.; Karna, S. P. Tryptophan-Gold Nanoparticle Interaction: A First-Principles Quantum Mechanical Study. J. Phys. Chem. C 2011, 115, 22818-22826.

(42) Hinterdorfer, P., van Oijen, A., Eds. Handbook of Single-Molecule Biophysics; SpringerVerlag: New York, 2009.

(43) Israelachvili, J. Intermolecular and Surface Forces; Elsevier, 2011.

(44) Lide, D. R. Handbook of Chemistry and Physics, 83rd ed.; CRC Press, 2002. 


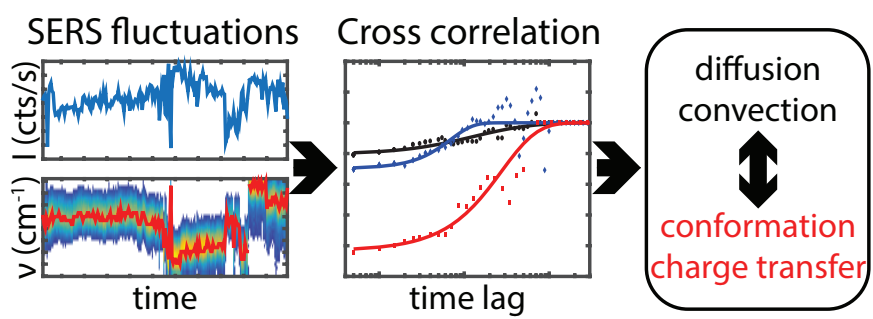

